\title{
Evaluation of Single-Tooth Implants in the Second Molar Region: A 5-Year Life- Table Analysis of a Retrospective Study
}

Ki-Tae Koo, * Ulf M.E. Wikesjö, † Jang-Yeol Park, * Tae-Il Kim, * Yang-Jo Seol, * Young Ku, * In-Chul Rhyu, ${ }^{*}$ Chong-Pyoung Chung, ${ }^{*}$ and Yong-Moo Lee*

Background: To our knowledge, no study has evaluated the success or survival rate of single-tooth implants that replaced missing maxillary and mandibular second molars. The purpose of the present study was to evaluate the 1 - to 5 -year cumulative survival rate (CSR) for single-tooth implants placed in the second molar region and the effects of associated factors.

Methods: Four hundred eighty-nine patients (298 males and 191 females; age range: 23 to 91 years; mean age: 47 years) who were admitted to the Seoul National University Dental Hospital between March 2003 and July 2008 and treated with single-tooth implants in the second molar region (227 maxillary implants and 294 mandibular implants; total: 521 implants) were included in the study. Thirty-two subjects received two implants each. A 1 - to 5-year CSR was calculated using a life-table analysis. A comparison of CSRs between maxillary versus mandibular implants, one-stage versus two-stage implants, short $(\leq 8.5 \mathrm{~mm})$ versus long $(>10 \mathrm{~mm})$ implants, and standard-diameter $(\leq 4.0 \mathrm{~mm})$ versus widediameter $(\geq 5.0 \mathrm{~mm})$ implants was performed using Wilcoxon (Gehan) statistics. $P<0.05$ was considered significant.

Results: Fifteen of the 521 implants were lost between insertion and the follow-up examinations. The 1 - to 5 -year CSR was $95.1 \%$. There were no statistically significant differences in CSRs between implants placed in maxillas and mandibles $(96.3 \%$ versus $94.9 \%$, respectively; $P=0.084)$, one- and two-stage implants $(95.6 \%$ versus $94.7 \%$, respectively; $P=$ $0.267)$, short and long implants (100\% versus $95.1 \%$, respectively; $P=0.582$ ), and standard- and wide-diameter implants (93.8\% versus $96.8 \%$, respectively; $P=0.065)$.

Conclusions: Within the limitations of the study, the placement of single-tooth implants in the second molar region was an effective and reliable treatment modality. Also, associated factors such as implant diameter, length, and location (the maxilla versus the mandible) may not have an impact on the longterm success of implants. J Periodontol 2010;81:1242-1249.

\section{KEY WORDS}

Dental implants, single-tooth; life tables; survival rate.

\footnotetext{
* Department of Periodontology, School of Dentistry, and Dental Research Institute, Seoul National University, Seoul, Korea.

$\dagger$ Laboratory for Applied Periodontal and Craniofacial Regeneration, Departments of Periodontics and Oral Biology, Medical College of Georgia, School of Dentistry, Augusta, GA.
}

I mplant dentistry appears an effective and predictable treatment modality for edentulous and partially edentulous patients. Over $80 \%$ of patients who receive implant treatment are partially edentulous. ${ }^{1}$ Also, the use of single-tooth implants to replace missing teeth has increased. A systematic review ${ }^{2}$ reported a success rate of $95.1 \%$ for single-tooth implant restorations at 5 years. Some studies $^{3,4}$ that focused on implants placed in the posterior mandible and maxilla also report high success or survival rates. To our knowledge, no study has evaluated the success or survival rate of singletooth implants that have replaced missing maxillary and mandibular second molars.

Controversy exists whether missing second molars should or should not be replaced. ${ }^{5,6}$ In case of a replacement, few restorative options are available. A fixed, partial denture does not appear a treatment of choice because cantilevered reconstructions have produced unfavorable biomechanics, and a removable partial denture may, at best, provide uncomfortable function and wearing. ${ }^{7}$ Consequently, the use of single-tooth implants for second molar reconstructions appear to be a preferred treatment. Although it may be argued that missing second molars should not be replaced, ${ }^{8}$ adverse consequences of not replacing second molars, such as the disturbance of a balanced occlusion,

doi: 10.1902/jop.2010.100064 
supra-eruption of the opposing dentition, and disharmony in chewing patterns, may argue in favor of reconstruction of missing second molars. ${ }^{9,10}$ The purpose of this study was to evaluate the long-term cumulative survival rate (CSR) for single-tooth implants placed in the second molar region and to evaluate the effect of associated factors including placement in the maxilla versus the mandible, using a one-stage protocol versus a two-stage protocol, and considering the diameter and length of the implant.

\section{MATERIALS AND METHODS}

\section{Patients}

Four hundred eighty-nine subjects (298 males and 191 females; age range: 23 to 91 years; mean age: 47 years) who were admitted to the Seoul National University Dental Hospital between March 2003 and July 2008 and treated with single-tooth implants in the second molar region were included in the study. A total of 521 external-hexed, single-tooth implants ${ }^{\dagger}$ (227 maxillary and 294 mandibular implants) with an anodizing oxidation surface ${ }^{\S}$ were used to replace missing second molars. Thirty-two subjects received two implants each. The preoperative evaluation consisted of a comprehensive periodontal examination, clinical photographic documentation, study casts, a radiographic evaluation, and a customized questionnaire to evaluate patients' systemic conditions, smoking habits, and parafunctions such as bruxism. A consultation was provided to each patient to thoroughly explain the surgical and prosthetic procedure, and a written consent form was obtained. The protocol was approved by the institutional review board (IRB) at Seoul National University Dental Hospital (IRB no. CRI09013) and was conducted in accordance with the Helsinki Declaration of 1975 as revised in 2000.

Exclusion criteria included uncontrolled medically compromised subjects, subjects with untreated active periodontitis, heavy smokers (>20 cigarettes/day), and subjects with bruxism or clenching habits. Moreover, subjects with inadequate bone volume were excluded. The bone height of the implant site had to be $\geq 7 \mathrm{~mm}$, which eliminated subjects in need of a sinus augmentation or crestal bone augmentation. Subjects were also excluded if bone grafting was performed in conjunction with implant placement. This criterion eliminated subjects who received immediate implant insertion after tooth extraction and bone grafting in conjunction with implant placement.

\section{Surgical Protocol}

Implant placement was performed strictly following the routines of Brånemark et al. ${ }^{11} \mathrm{~A}$ two-stage surgical protocol was used when poor quality (types III and IV) bone was encountered. In cases of dense bone quality (types I and II), commercially available heal- ing abutments ${ }^{\|}$were connected at the time of surgery. A total of 231 and 290 implants using the one-stage and two-stage protocols, respectively, were placed. The bone density at the implant sites was determined according to the tactile approach described by Lekholm et al. ${ }^{12}$

\section{Prosthodontic Protocol}

Uncovering of the implants placed using the twostage protocol was performed after a healing period of 3 to 4 months in the mandible and 6 months in the maxilla. A healing period of 3 to 4 weeks was allowed until the prosthetic abutments were connected. Commercially available abutment screws ${ }^{\#}$ were tightened with a torque wrench to $30 \mathrm{Ncm}$, and this was repeated 10 minutes later. No attempts were made to splint the single-tooth implants to a natural tooth. Periapical radiographs using the parallel-cone technique were obtained presurgery, immediately post-surgery, after prosthetic abutment connection, and at follow-up appointments. Subjects returned every 3 months for a detailed maintenance including cleaning, oral hygiene reinforcement, and a radiographic evaluation for the first year. Annual follow-ups were scheduled thereafter.

\section{Statistical Analyses}

The 1 - to 5-year CSR was calculated using life-table analysis. The criteria for success were as follows: the absence of clinically detectable implant mobility, absence of pain or subjective sensation, absence of recurrent peri-implant infection, and absence of continuous radiolucency around the implant. ${ }^{13,14}$ Also, comparisons in CSRs between maxillary versus mandibular, one-stage versus two-stage, short $(\leq 8.5 \mathrm{~mm})$ versus long $(>10 \mathrm{~mm})$, and standard-diameter $(\leq 4.0$ $\mathrm{mm}$ ) versus wide-diameter $(\geq 5.0 \mathrm{~mm})$ implants were performed using Wilcoxon (Gehan) statistics. $P<0.05$ was considered significant.

\section{RESULTS}

\section{Implant Distribution}

Table 1 shows the distribution of implants grouped by bone quality, diameter, and length. Of the $521 \mathrm{im}-$ plants, $44 \%$ were placed in type II bone, $39 \%$ were placed in type III bone, and $17 \%$ were placed in type IV bone. Contrasting maxillary to mandibular sites, respectively, 32\% (73 sites) versus 52\% (154 sites) exhibited type II bone, 40\% (91 sites) versus 39\% (113 sites) exhibited type III bone, and 28\% (63 sites) versus $9 \%$ (27 sites) exhibited type IV bone. Fifty-six percent of the implants were standard-diameter implants,

\footnotetext{
‡ Mark III, Nobel Biocare, Gothenburg, Sweden.

$\S$ Ti-Unite, Nobel Biocare.

II Nobel Biocare.

II Nobel Biocare.

\# Nobel Biocare.
} 
Table I.

Distribution of Implants Relative to Length $(\mathrm{mm})$ and Diameter $(\mathrm{mm})$ and to Bone Quality (types II, III, and IV)

\begin{tabular}{|c|c|c|c|c|c|c|c|c|c|c|c|c|c|}
\hline \multirow[b]{2}{*}{ Length } & \multirow[b]{2}{*}{$n(\%)$} & \multicolumn{4}{|c|}{ Diameter: 3.75} & \multicolumn{4}{|c|}{ Diameter: 4.0} & \multicolumn{4}{|c|}{ Diameter: 5.0} \\
\hline & & \multicolumn{3}{|c|}{ Type II Type III Type IV } & \multirow[t]{2}{*}{ Total } & \multicolumn{3}{|c|}{ Type II Type III Type IV } & \multirow[t]{2}{*}{ Total } & \multicolumn{3}{|c|}{ Type II Type III Type IV } & \multirow[t]{2}{*}{ Total } \\
\hline Maxilla & & & & & & & & & & & & & \\
\hline 7 & $0(0)$ & 0 & 0 & 0 & 0 & 0 & 0 & 0 & 0 & 0 & 0 & 0 & 0 \\
\hline 8.5 & $49(22)$ & 1 & 0 & 0 & I & 13 & 12 & 0 & 25 & 11 & 9 & 3 & 23 \\
\hline 10 & $103(45)$ & 0 & 0 & 0 & 0 & 15 & 21 & 20 & 56 & II & 20 & 16 & 47 \\
\hline 11.5 & $68(30)$ & 0 & 0 & 0 & 0 & 8 & 14 & 16 & 38 & 7 & 15 & 8 & 30 \\
\hline 13 & $7(3)$ & 0 & 0 & 0 & 0 & 3 & 0 & 0 & 3 & 4 & 0 & 0 & 4 \\
\hline Total & $227(100)$ & 1 & 0 & 0 & I (0.4\%) & 39 & 47 & 36 & $122(54 \%)$ & 33 & 44 & 27 & 104 (46\%) \\
\hline \multicolumn{14}{|l|}{ Mandible } \\
\hline 7 & $7(2)$ & 0 & 0 & 0 & 0 & । & । & 0 & 2 & 5 & 0 & 0 & 5 \\
\hline 8.5 & $66(23)$ & 0 & I & 0 & I & 8 & 16 & 6 & 30 & 20 & 13 & 2 & 35 \\
\hline 10 & $113(38)$ & I & 2 & 0 & 3 & 27 & 34 & 2 & 63 & 29 & 13 & 5 & 47 \\
\hline 11.5 & $87(30)$ & 1 & 0 & 0 & । & 27 & 20 & 4 & 51 & 24 & 7 & 4 & 35 \\
\hline 13 & $21(7)$ & 0 & 0 & 0 & 0 & 8 & 6 & 3 & 17 & 3 & 0 & I & 4 \\
\hline Total & $294(100)$ & 2 & 3 & 0 & $5(2 \%)$ & 71 & 77 & 15 & 163 (55\%) & 81 & 33 & 12 & $126(43 \%)$ \\
\hline $\begin{array}{l}\text { Total maxilla } \\
\text { and mandible }\end{array}$ & $521(100)$ & 3 & 3 & 0 & $6(1 \%)$ & 110 & 124 & 51 & 285 (55\%) & 114 & 77 & 39 & $230(44 \%)$ \\
\hline
\end{tabular}

Table 2.

\section{Life-Table Analysis of Implant Survival}

\begin{tabular}{lcccc}
\hline Interval & $\begin{array}{c}\text { Implants }(n) \text { at Start } \\
\text { of Interval }\end{array}$ & $\begin{array}{c}\text { Implant Failures }(n) \\
\text { During Interval }\end{array}$ & Interval Survival (\%) & CSR (\%) \\
\hline Placement to loading & 521 & 2 & 99.6 & 99.6 \\
0 to I years & 512 & 5 & 99.0 & 98.6 \\
I to 2 years & 440 & 2 & 99.5 & 98.1 \\
2 to 3 years & 343 & 3 & 99.0 & 97.1 \\
3 to 4 years & 281 & 1 & 99.6 & 96.8 \\
4 to 5 years & 236 & 2 & 98.3 & 95.1 \\
\hline
\end{tabular}

and $58 \%$ of these were placed in the mandible. For the wide-diameter implants, 55\% were placed in the mandible. Twenty-three percent of the implants were short implants, and $60 \%$ were placed in the mandible.

\section{Life-Table Analysis}

Of 521 implants, 15 implants were lost between insertion and the 4- to 5-year follow-up examinations (Table 2). Two implants were lost prior to loading, and the biggest loss was observed within the first year of loading during which five implants failed. The CSR at 5 years was $95.1 \%$.

\section{CSRs for Maxillary Versus Mandibular Implants}

The CSR for implants placed in the maxilla was $96.3 \%$ compared to $94.9 \%$ for implants placed in the mandible $(P=0.084$; Table 3$)$. For implants placed in the maxilla, one implant was lost within 1 year of loading whereas two implants failed in the 2- to 3-year interval, and another two implants failed in the 4- to 5 -year intervals. For implants placed in the mandible, the biggest implant loss was observed between insertion and 1 year of loading during which six implants failed. Two implants failed prior to loading, whereas four implants were lost within 1 year of loading. 
Table 3.

\section{Distribution of One- and Two-Stage Implants}

\begin{tabular}{lcccc}
\hline Distribution & One-Stage $(n)$ Two-Stage $(n)$ & Total/CSR (\%) \\
\hline Maxilla & 68 & 159 & $227 / 96.3$ \\
Mandible & 163 & 131 & $294 / 94.9$ \\
Total/CSR (\%) & $231 / 95.6$ & $290 / 94.7$ & $P=0.084 / P=0.267$ \\
\hline
\end{tabular}

\section{CSRs for One-Stage Protocol Versus Two-Stage Protocol}

For the 231 implants placed as a one-stage procedure, 226 implants survived, which yielded a CSR of $95.6 \%$. For the 290 implants placed as a two-stage procedure, 280 implants survived, which yielded a CSR of $94.7 \%$. There were no statistically significant differences in CSRs between one-stage versus twostage protocols $(P=0.267)$.

\section{CSRs for Short ( $\leq 8.5 \mathrm{~mm})$ Versus Long (>10 mm) Implants}

All short implants survived, as no implant loss was observed between insertion and the 4- to 5-year follow-up examinations. The survival rate for long implants was $95.1 \%$. There were no statistically significant differences in CSRs between short versus long implants $(P=0.582)$.

\section{CSRs for Standard-Diameter (3.75 and $4.0 \mathrm{~mm}$ ) Versus Wide-Diameter $(5.0 \mathrm{~mm})$ Implants}

Eleven standard-diameter implants failed, whereas four implant failures were observed among the widediameter implants. Six standard-diameter implants were lost between insertion and the 0 - to 1 -year follow-up examination, whereas two implants failed 1 to 2 years after loading, two implants failed 2 to 3 years after loading, and one implant failed 4 to 5 years postloading. For the wide-diameter implants, one failure was observed in each of the following intervals; 0 to 1,2 to 3,3 to 4 , and 4 to 5 years after loading. The CSR for standard-diameter implants was $93.8 \%$ compared to $96.8 \%$ for wide-diameter implants, without statistically significant differences between standard- and wide-diameter implants $(P=0.065)$.

\section{Implant Failures}

Descriptions of failed implants are shown in Table 4. Of the 15 failed implants, 10 implants were placed in the mandible. The majority of failed implants were standard-diameter $(\mathrm{n}=11)$ with a length $>10 \mathrm{~mm}$.

\section{Table 4.}

\section{Description of Failed Implants}

\begin{tabular}{|c|c|c|c|c|c|}
\hline Arch & Duration & Diameter $(\mathrm{mm})$ & Length (mm) & Stage & Bone Quality (type) \\
\hline Maxilla & 0 to 1 years & 4.0 & 10 & 2 & III \\
\hline Maxilla & 2 to 3 years & 4.0 & 11.5 & 2 & IV \\
\hline Maxilla & 2 to 3 years & 5.0 & 10 & I & $\|$ \\
\hline Maxilla & 4 to 5 years & 5.0 & 10 & । & $\|$ \\
\hline Maxilla & 4 to 5 years & 4.0 & 10 & 2 & ॥11 \\
\hline Mandible & Placement to loading & 4.0 & 13 & 2 & III \\
\hline Mandible & Placement to loading & 4.0 & 10 & 2 & III \\
\hline Mandible & 0 to 1 years & 4.0 & 11.5 & । & $\|$ \\
\hline Mandible & 0 to I years & 4.0 & 13 & 2 & IV \\
\hline Mandible & 0 to I years & 4.0 & 13 & 2 & IV \\
\hline Mandible & 0 to 1 years & 5.0 & 10 & 2 & III \\
\hline Mandible & I to 2 years & 4.0 & 11.5 & । & $\|$ \\
\hline Mandible & I to 2 years & 4.0 & 10 & 2 & IV \\
\hline Mandible & 2 to 3 years & 4.0 & 11.5 & I & $\|$ \\
\hline Mandible & 3 to 4 years & 5.0 & 10 & 2 & IV \\
\hline
\end{tabular}




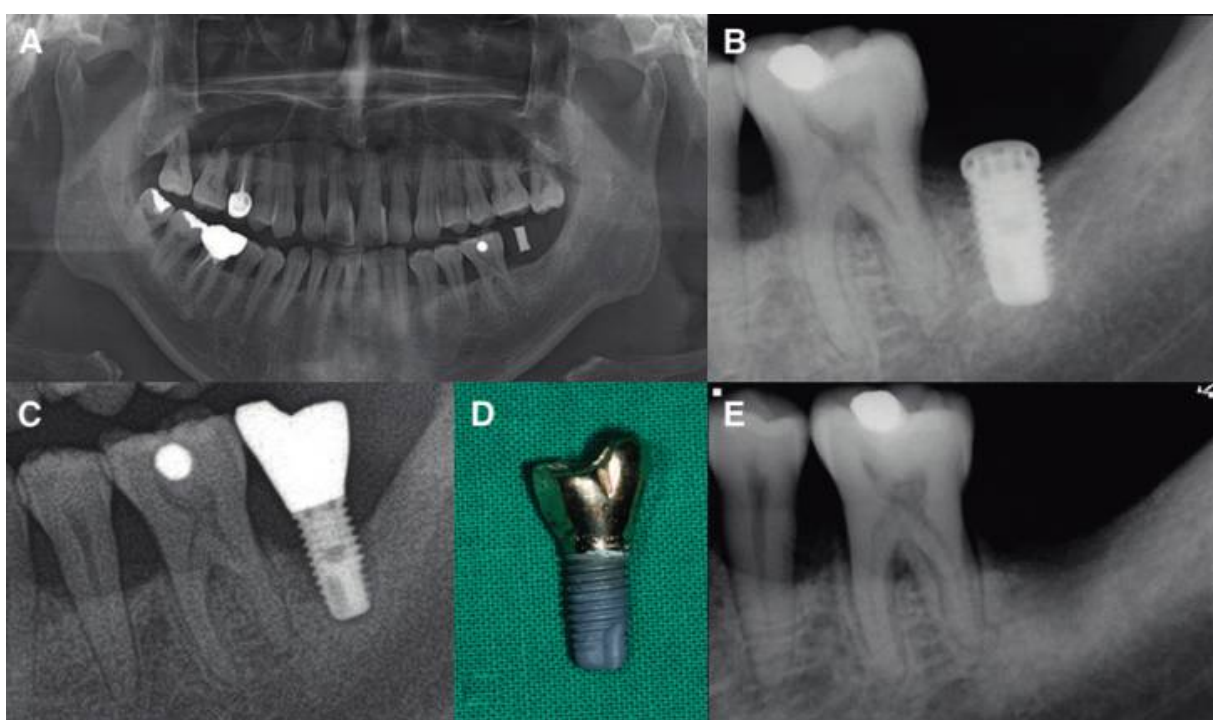

Figure I.

Clinical presentation of a wide-diameter implant placed in the mandible as a two-stage procedure showing symptoms of infection within I year of loading. A) Pretreatment panoramic radiograph of the edentulated left mandible, second molar site. B) Radiograph obtained immediately after implant placement. C) Radiograph showing radiolucencies surrounding the implant. D) Photograph of the removed failed implant. E) Radiograph showing healing at 4 weeks after implant removal.

Four wide-diameter implants with a length of $10 \mathrm{~mm}$ failed. Most of the failures occurred between placement and within 2 years of loading, as nine of 15 failures occurred during this time period. Three onestage implants placed in the mandible failed, whereas two one-stage implants were lost in the maxilla. All five one-stage implants were placed in type II bone. Ten two-stage implants failed with poor bone quality and stability as the main reason for the loss. All twostage implants were placed in type III or IV bone. One wide-diameter implant placed in type III bone as a twostage procedure showed acute symptoms of infection within 1 year after loading and was removed (Fig. 1). Of the 15 failed implants, five implants were placed in type IV bone, five implants were placed in type III bone, and five implants were placed in type II bone, respectively.

\section{DISCUSSION}

The present study evaluates the CSR for single-tooth implants that replaced missing second molar teeth. The CSR was $95.1 \%$ at 5 years after loading. This result is meaningful because, to our knowledge, no previous study has specifically focused on the second molar region. However, a large body of evidence evaluating survival rates of single-tooth implants has suggested favorable outcomes. In a review, Goodacre et al. ${ }^{15}$ reported a survival rate of $97 \%$ for single-tooth implants. Meta-analyses by Lindh et al. ${ }^{16}$ and Jung et al. ${ }^{17}$ reported a CSR of $97.5 \%$ and $94.5 \%$, respectively. Nevertheless, the implant position was not con- sidered in these reports, and only a few studies have focused on posterior single-tooth implants. Becker and Becker ${ }^{3}$ first published a report concerning the survival rate of posterior single-tooth implants and observed a CSR of 95\%. Misch et al. ${ }^{4}$ reported a survival rate of $98.9 \%$ from a 10 -year retrospective study on posterior single-tooth implants. A systematic review ${ }^{2}$ estimated that the prosthetic success rate at 5 years after loading of implants was $95.1 \%$. However, immediate comparisons with the present study may not be meaningful because single-tooth implants that replaced first molars were also included in these studies.

In the present study, implants placed in both the maxilla and mandible were evaluated. Two hundred twenty-seven implants were placed in the maxilla and yielded a CSR of $96.3 \%$, whereas the CSR for 294 mandibular implants was $94.9 \%$. Although there was a slight difference in the number of implants placed, no statistically significant differences in CSRs existed between the groups. This may be of interest because it is normally perceived that implants placed in the mandible are more successful than those placed in the maxilla. In a long-term prospective study by Becker et al., ${ }^{18}$ the CSR of single-tooth molar implants placed in the mandible was $91.5 \%$ compared to $82.9 \%$ for the maxilla. An 8 -year longitudinal study ${ }^{1}$ reported success rates for hollow-basket and plasma-sprayed implants that replaced maxillary and mandibular molars of $86.7 \%$ and $95.4 \%$, respectively. van Steenberghe et al. ${ }^{19}$ placed 27 implants in the posterior maxilla and 171 implants in the mandible and lost one maxillary and six mandibular implants. Balshi et al. ${ }^{20}$ evaluated multiple implants that replaced maxillary and mandibular molars and reported a $100 \%$ success rate for maxillary implants and a $95.9 \%$ success rate for mandibular implants. These contrasting results imply that maxillary implants are not always unfavorable, and a more discrete parameter such as bone quality is necessary for meaningful comparisons. In the present study, none of the implants were placed in type I bone, and the majority of the implants were placed in type II or III bone. The overall percentage of bone sites of types II and III was $72 \%$ for the maxilla and $91 \%$ for the mandible, respectively. The remaining $28 \%$ and 
$9 \%$ were of type IV bone for the maxilla and mandible, respectively. From these observations, it appears that bone quality did not affect the outcomes of the present study, although it was reported that poor bone quality renders an increased risk for implant failure. ${ }^{21}$ Also, despite the higher percentage of type IV bone, only five implants in the maxilla failed, whereas 10 failures occurred in the mandible suggesting that favorable outcomes might be expected in the maxilla as well.

The present study also evaluates the CSR for implants placed in a one-stage procedure versus a two-stage procedure. For the one-stage group, five of 231 implants failed, yielding a CSR of $95.6 \%$. For the two-stage group, 10 of 290 implants failed, yielding a CSR of $94.7 \%$. There was no statistically significant difference between the groups. This result concurs with a number of studies ${ }^{22-25}$ that showed similar results irrespective of whether a submerged protocol was applied or not. However, in the present study, one-stage implants were placed in type I or II bone, whereas two-stage implants were placed in type III or IV bone. This variability in bone conditions between the two groups limits a truly meaningful comparison, although within the framework of this study, controlling for bone quality in the two groups was difficult because of the small number of patients. Another shortcoming that deserves attention is the fact that only 68 of 231 one-stage implants were placed in the maxilla compared to 159 of 290 two-stage implants. These mismatches may have impacted the results; thus, additional studies using a larger sample size and more equal distribution among bone qualities and maxillary versus mandibular sites may cast further insight to confirm or reject the observations in the present study.

Comparisons of CSRs in the present study between the short versus long and standard-diameter versus wide-diameter implants corroborate the results reported by Jung et al. ${ }^{10}$ Both studies suggested that no significant differences in survival rates should be expected for short versus long implants, which in the present study amounted to $100 \%$ versus $95.1 \%$ and to $93.8 \%$ versus $96.8 \%$ for standard-diameter versus wide-diameter implants, respectively. It is noteworthy how well the short implants performed, despite the anatomic and biomechanical challenges in the second molar region. However, the small sample size in the present study and because only $23 \%$ ( $40 \%$ in the maxilla) of the implants were short implants may preclude definitive conclusions.

Controversy still exists whether wide-diameter implants should be preferred in the posterior region. ${ }^{26,27}$ In the present study, $56 \%$ of 521 implants placed were standard-diameter implants, including $42 \%$ placed in the maxilla and $58 \%$ placed in the mandible. This distribution was similar for wide-diameter implants as $45 \%$ were placed in the maxilla, and $55 \%$ were placed in the mandible. Of the standard-diameter implants, $39 \%, 44 \%$, and $17 \%$ were placed in type II, III, and IV bone, respectively, versus $50 \%, 33 \%$, and $17 \%$ of the wide-diameter implants that were placed in type II, III, and IV bone, respectively. Although there were minor differences in the percentages of bone sites of types II, III, and IV, the difference in CSRs between the groups was not statistically significant. The two wide-diameter implants that failed were placed in type III and type IV bone in the mandible, and two implants failed in type II bone in the maxilla. These results corroborated the observations presented by Friberg et al. ${ }^{27}$ and suggest that implant diameter is not a determining factor for implant success. Moreover, within the limitations of the present study, standard-diameter, single-tooth implants may function as equally well as wide-diameter, single-tooth implants even in challenging situations as in the second molar region.

Even though a 5-year CSR of $95.1 \%$ in the present study appears high, several recent studies ${ }^{28-30}$ analyzed the midterm success of dental implants and reported higher survival/success rates. In a 5-year report by Sullivan et al., ${ }^{28}$ early loading ( 2 months) of 526 implants** with a dual thermal acid-etched surface presented a CSR of $97.9 \%$ at 5 years. Cochran et al. ${ }^{29}$ reported a CSR of $99.26 \%$ at 5 years using sandblasted acid-etched (SLA) implants. ${ }^{\dagger \dagger}$ Another study using the SLA surface showed a 5-year success rate of $99 \%$ with 104 implants inserted into posterior sites of 51 partially edentulous patients. ${ }^{30}$ Comparing these results with those of the present study, the reason for the difference may be the result of different surface treatments. However, a careful interpretation must be exercised because the location and conditions of the implants were quite different. Although the loading conditions were more favorable for the present study with healing periods of 12 to 24 weeks, only single implants in the second molar region were evaluated, whereas in the study by Sullivan et al. ${ }^{28}$ with healing of 6 to 8 weeks, $23 \%$ of the implants were placed in the anterior segments, and only 118 of 526 implants were single implants. Also, in the study by Bornstein et al., ${ }^{30}$ only 39 implants were restored with a single crown, whereas the remaining 64 implants were either splinted or used as an abutment for implant-supported, fixed, partial dentures. Cochran et al. $^{29}$ did not report whether the implants were placed in the anterior or posterior section of the mouth and whether they were single crowns or splinted. Considering that a single implant could be disadvantageous, and the force of occlusion increases toward the posterior area, especially in the second molar region, ${ }^{10}$ it is difficult to conclude that the surface

* * Osseotite, Biomet 3i, Palm Beach Gardens, FL.

$\dagger \dagger$ Straumann SLA, Institute Straumann, Waldenburg, Switzerland. 
treatment affected the outcomes and that the results of the present study are less favorable than comparable reports. Also, the exclusion criterion for heavy smokers was more lenient in the present study. Patients who smoked $>20$ cigarettes per day were considered heavy smokers, whereas in comparable studies, ${ }^{28,29}$ the standard was $>10$ cigarettes per day. It is difficult to directly correlate the quantity of cigarettes smoked per day to implant success. However, because elevated rates of implant failures are associated with heavy smoking, ${ }^{31}$ a more strict analysis of risk profiles and informing patients accordingly during treatment planning are recommended.

\section{CONCLUSION}

Within the limitations of this study, the results indicate that the placement of single-tooth implants in the second molar region is an effective and reliable treatment modality.

\section{ACKNOWLEDGMENTS}

No external funding, apart from the support of the authors' institutions, was available for this study. The authors report no conflict of interest related to this study.

\section{REFERENCES}

1. Buser D, Mericske-Stern R, Bernard JP, et al. Longterm evaluation of non-submerged ITI implants. Part 1: 8-year life table analysis of a prospective multi-center study with 2,359 implants. Clin Oral Implants Res 1997;8:161-172.

2. Salinas TJ, Eckert SE. In patients requiring single-tooth replacement, what are the outcomes of implant- as compared to tooth-supported restorations? Int J Oral Maxillofac Implants 2007;22(Suppl.):71-95 (erratum 2008;23:56).

3. Becker W, Becker BE. Replacement of maxillary and mandibular molars with single endosseous implant restorations: A retrospective study. J Prosthet Dent 1995;74:51-55.

4. Misch CE, Misch-Dietsh F, Silc J, Barboza E, Cianciola LJ, Kazor C. Posterior implant single-tooth replacement and status of adjacent teeth during a 10-year period: A retrospective report. JPeriodontol 2008;79:2378-2382.

5. Kayser AF. The shortened dental arch: A therapeutic concept in reduced dentitions and certain high-risk groups. Int J Periodontics Restorative Dent 1989;9: 426-449.

6. Witter DJ, van Elteren P, Kayser A. Signs and symptoms of mandibular dysfunction in shortened dental arches. J Oral Rehabil 1988;15:413-420.

7. Himmel R, Pilo R, Assif D, et al. The cantilever fixed partial denture - A literature review. J Prosthet Dent 1992;67:484-487.

8. Shugars DA, Bader JD, Phillips SW Jr., White BA, Brantley CF. The consequences of not replacing a missing posterior tooth. J Am Dent Assoc 2000;131: 1317-1323.

9. Shillingburg HT Jr., Hobo S, Whitsett LD. Fundamentals of Fixed Prosthodontics, 3rd ed. Chicago: Quintessence; 1997:85.
10. Jung UW, Choi JY, Kim C, et al. Evaluation of mandibular posterior single implants with two different surfaces: A 5-year comparative study. J Periodontol 2008;79:1857-1863.

11. Brånemark PI, Hansson BO, Adell R, et al. Oseeointegrated implants in the treatment of the edentulous jaw. Experience from a 10-year period. Scand J Plast Reconstr Surg Suppl 1977;16:1-132.

12. Lekholm U, Zarb GA, Albrektsson T, eds. TissueIntegrated Prostheses: Osseointegration in Clinical Dentistry. Chicago: Quintessence; 1985:199-220.

13. Buser D, von Arx T, ten Bruggenkate C, Weingart D. Basic surgical principles with ITI implants. Clin Oral Implants Res 2000;11(Suppl. 1):59-68.

14. Cochran DL, Buser D, ten Bruggenkate CM, et al. The use of reduced healing times on ITI implants with a sandblasted and acid-etched (SLA) surface: Early results from clinical trials on ITI SLA implants. Clin Oral Implants Res 2002;13:144-153.

15. Goodacre CJ, Bernal G, Rungcharassaeng K, Kan JY. Clinical complications with implants and implant prostheses. J Prosthet Dent 2003;90:121-132.

16. Lindh T, Gunne J, Tillberg A, Molin M. A meta-analysis of implants in partial edentulism. Clin Oral Implants Res 1998;9:80-90.

17. Jung RE, Pjetursson BE, Glauser R, Zembic A, Zwahlen M, Lang NP. A systematic review of the 5-year survival and complication rates of implant-supported single crowns. Clin Oral Implants Res 2008;19:119-130.

18. Becker W, Becker BE, Al-Suwyed A, Al-Mubarak S. Long-term evaluation of 282 implants in maxillary and mandibular molar positions: A prospective study. J Periodontol 1999;70:896-901.

19. van Steenberghe D, Lekholm U, Bolender C, et al. Applicability of osseointegrated oral implants in the rehabilitation of partial edentulism: A prospective multicenter study on 558 fixtures. Int J Oral Maxillofac Implants 1990;5:272-281.

20. Balshi TJ, Hernandez RE, Pryszlak MC, Rangert B. A comparative study of one implant versus two replacing a single molar. Int J Oral Maxillofac Implants 1996; 11:372-378.

21. Jaffin RA, Berman CL. The excessive loss of Brånemark fixtures in type IV bone: A 5-year analysis. J Periodontol 1991;62:2-4.

22. Engquist B, Astrand P, Anzen B, et al. Simplified methods of implant treatment in the edentulous lower jaw: A 3-year follow-up report of a controlled prospective study of one-stage versus two-stage surgery and early loading. Clin Implant Dent Relat Res 2005;7:95-104.

23. Becktor JP, Isaksson S, Billström C. A prospective multicenter study using two different surgical approaches in the mandible with turned Brånemark implants: Conventional loading using fixed prosthesis. Clin Implant Dent Relat Res 2007;9:179-185.

24. Friberg B, Raghoebar G, Grunert I, Hobkirk JA, Tepper G. A 5-year prospective multicenter study on 1-stage smooth-surface Brånemark system implants with early loading in edentulous mandibles. Int $J$ Oral Maxillofac Implants 2008;23:481-486.

25. Esposito M, Grusovin MG, Chew YS, Coulthard P, Worthington HV. Interventions for replacing missing teeth: 1 -versus 2-stage implant placement. Cochrane Database Syst Rev 2009;(3):CD006698.

26. Degidi M, Piattelli A, lezzi G, Carinci F. Wide-diameter implants: Analysis of clinical outcome of 304 fixtures. J Periodontol 2007;78:52-58. 
27. Friberg B, Ekestubbe A, Sennerby L. Clinical outcome of Brånemark System implants of various diameters: A retrospective study. Int $J$ Oral Maxillofac Implants 2002;17:671-677.

28. Sullivan D, Vincenzi G, Feldman S. Early loading of Osseotitie implants 2 months after placement in the maxilla and mandible: A 5-year report. Int $J$ Oral Maxillofac Implants 2005;20:905-912.

29. Cochran D, Oates T, Morton D, Jones A, Buser D, Peters F. Clinical field trial examining an implant with a sand-blasted, acid-etched surface. J Periodontol 2007;78:974-982.

30. Bornstein MM, Schmid B, Belser UC, Lussi A, Buser D. Early loading of non-submerged titanium implants with a sandblasted and acid-etched surface. 5-year results of a prospective study in partially edentulous patients. Clin Oral Implants Res 2005;16:631-638.

31. Bain CA, Moy PK. The association between the failure of dental implants and cigarette smoking. Int J Oral Maxillofac Implants 1993;8:609-615.

Correspondence: Dr. Yong-Moo Lee, Department of Periodontology, School of Dentistry, Seoul National University, 28 Yongon-Dong, Chongno-Ku, Seoul 110-749, Korea. Fax: 82-2-744-0051; e-mail: ymlee@snu.ac.kr.

Submitted February 3, 2010; accepted for publication March 24, 2010. 